\title{
DIFFERENTIABLE SELECTION OF OPTIMAL SOLUTIONS IN PARAMETRIC LINEAR PROGRAMMING
}

\author{
DINH THE LUC AND PHAM HUY DIEN
}

(Communicated by Joseph S. B. Mitchell)

\begin{abstract}
In the present paper we prove that if the data of a parametric linear optimization problem are smooth, the solution map admits a local smooth selection "almost" everywhere. This in particular shows that the set of points where the marginal function of the problem is nondifferentiable is nowhere dense.
\end{abstract}

\section{IntRoduction}

Subdifferential calculus for marginal functions defined by parametric optimization problems has recently become a subject of much attention because of its potential applications to the solution of various questions arising in optimization, control theory and other areas of applied mathematics. "Outer" estimation for the subdifferentials of marginal functions has been studied in numerous papers (see e.g. [1], [10], [17], [19], [21]). However, how to calculate at least one element from these subdifferentials remains a difficult part of nonsmooth optimization (see [6], [9], [12], $[20])$. In most existing results an important condition to require is that the solution map admits a locally Lipschitz selection, which is differentiable at a point of our consideration.

Motivated by the above condition, we set our aim to investigate differentiability properties of the marginal function and the solution map of a parametric linear optimization problem. The main result of the paper states that if the objective and the constraints of the problem are smooth, there is an open dense set in the parameter space where the feasible set is continuous (Section 2) and the solution map admits a smooth local selection (Section 3).

This result is remarkable in part for the following reason. It is known (Michael's selection theorem [2]) that under suitable conditions, a set-valued map admits a continuous (or Lipschitz continuous) selection. We have a natural question: do differentiable selections exist? The result of Section 3 provides a sufficient condition for a positive answer.

Received by the editors March 28, 1994 and, in revised form, September 13, 1994.

1991 Mathematics Subject Classification. Primary 90C31; Secondary 90C05, 49K40.

This work was supported in part by the Program on Applied Mathematics and was completed during the authors' stay at the Laboratory for Applied Mathematics, University of Pau, France. 


\section{STABILITY OF LINEAR SYSTEMS}

Throughout the paper we shall make use of the following notation: $p, q, r, s$ are positive integers; $c, a_{1}, \ldots, a_{p+q}$ denote continuous vector functions from $R^{r}$ to $R^{s}$; $\alpha_{1}, \ldots, \alpha_{p+q}$ stand for continuous scalar functions on $R^{r}$; and $\langle\cdot, \cdot\rangle$ stands for the inner product in $R^{s}$. The marginal function we are going to study is defined as

$$
m(\omega):=\min \{\langle c(\omega), x\rangle \mid x \in M(\omega)\},
$$

where

$$
\begin{aligned}
M(\omega):=\left\{x \in R^{s} \mid\right. & \left\langle a_{i}(\omega), x\right\rangle+\alpha_{i}(\omega) \leq 0, i=1, \ldots, p, \\
\left\langle a_{j}(\omega), x\right\rangle+\alpha_{j}(\omega) & =0, j=p+1, \ldots, p+q\},
\end{aligned}
$$

and the solution map is defined by

$$
S(\omega):=\{x \in M(\omega) \mid\langle c(\omega), x\rangle=m(\omega)\} .
$$

By a selection of the solution map we mean any (single-valued) function $g(\omega)$ from $R^{r}$ to $R^{s}$ with $g(\omega) \in S(\omega)$ for all $\omega \in R^{r}$. Let us recall that a set-valued map $A$ from $R^{r}$ to $R^{s}$ is said to be closed at a point $\omega_{0} \in R^{r}$ if, whenever a sequence $\left\{\left(\omega_{n}, x_{n}\right)\right\} \subseteq R^{r} \times R^{s}$ with $x_{n} \in A\left(\omega_{n}\right)$ converges to $\left(\omega_{0}, x_{0}\right)$, one has $x_{0} \in A\left(\omega_{0}\right)$. $A$ is said to be lower continuous at $\omega_{0} \in R^{r}$ if for every neighborhood $U$ in $R^{s}$ with $A\left(\omega_{0}\right) \cap U \neq \emptyset$ there exists a neighborhood $W$ of $\omega_{0}$ in $R^{r}$ such that $A(\omega) \cap U \neq \emptyset$ for all $\omega \in W$ (here we use "lower continuous" instead of "lower semicontinuous" to avoid the confusion with lower semicontinuity of point-valued real functions). The map $A$ is said to be continuous at $\omega_{0}$ (on a nonempty set $W \subseteq R^{r}$ ) if it is closed and lower continuous at $\omega_{0}$ (at every point of $W$, resp.). There exist different definitions of continuity in the literature (see [2], [3], [4], [11], [14]). However, they are all equivalent to the one given above if the values of $A$ are compact in a bounded set. In this event, $A$ is continuous at $\omega_{0}$ if and only if the Hausdorff distance $h\left(A(\omega), A\left(\omega_{0}\right)\right)$ between $A(\omega)$ and $A\left(\omega_{0}\right)$ tends to 0 as $\omega$ tends to $\omega_{0}$. As to the concept of upper (semi-)continuity in the case of noncompact values, we do not pay our attention because in that case generally the map $M$ is never upper continuous. We shall adopt the convention that $A$ is continuous at $\omega_{0}$ with $A\left(\omega_{0}\right)$ empty if $A(\omega)$ is empty for all $\omega$ in some neighborhood of $\omega_{0}$.

Denote

$$
\begin{aligned}
& P(\omega):=\left\{x \in R^{s} \mid\left\langle a_{i}(\omega), x\right\rangle+\alpha_{i}(\omega) \leq 0, i=1, \ldots, p\right\} \\
& Q(\omega):=\left\{x \in R^{s} \mid\left\langle a_{j}(\omega), x\right\rangle+\alpha_{j}(\omega)=0, j=p+1, \ldots, p+q\right\} .
\end{aligned}
$$

Furthermore, define, for any subset $I$ of $\{1, \ldots, p\}$,

$$
\begin{aligned}
P_{I}(\omega) & :=\left\{x \in R^{s} \mid\left\langle a_{i}(\omega), x\right\rangle+\alpha_{i}(\omega) \leq 0, i \in I\right\}, \\
\widehat{P}_{I}(\omega) & :=\left\{x \in R^{s} \mid\left\langle a_{i}(\omega), x\right\rangle+\alpha_{i}(\omega)<0, i \in I\right\}, \\
\widehat{P}^{I}(\omega): & =\left\{x \in R^{s} \mid\left\langle a_{i}(\omega), x\right\rangle+\alpha_{i}(\omega)<0, i \in\{1, \ldots, p\} \backslash I\right\},
\end{aligned}
$$

and, for any subset $J$ of $\{1, \ldots, p+q\}$,

$$
Q_{J}(\omega):=\left\{x \in R^{s} \mid\left\langle a_{j}(\omega), x\right\rangle+\alpha_{j}(\omega)=0, j \in J\right\} .
$$

To date important results have been obtained on the continuity of the map $M(\cdot)$ (see for instance [3], [8], [15]). However, the following seems to be new. 
Proposition 2.1. For every open set $W_{0} \subseteq R^{r}$, there exists an open subset $W \subseteq$ $W_{0}$ such that $M$ is continuous on $W$. Consequently, the set of discontinuity of $M$ is nowhere dense.

To prove this proposition the following lemmas are needed.

Lemma 2.1. Let $\omega_{0}$ be a fixed point in $R^{r}$. Assume that the system $\left\{a_{j}\left(\omega_{0}\right), j \in J\right\}$ is linearly independent. Then, there exists a neighborhood $W$ of $\omega_{0}$ in $R^{r}$ such that the set-valued map $\omega \mapsto Q_{J}(\omega)$ is continuous on $W$.

Proof. It is clear that $Q_{J}(\cdot)$ is a closed map (which is true without the linear independence assumption). The lower continuity can be derived from Robinson's general criterion $([15])$, however we provide here a direct proof. Let $\nu:=|J|$ and $A\left(\omega_{0}\right)$ denote the $(\nu \times s)$-matrix whose rows are $a_{j}\left(\omega_{0}\right), j \in J$. It is evident that $\nu \leq s$ and $A\left(\omega_{0}\right)$ possesses a nonsingular $(\nu \times \nu)$-submatrix, say $C\left(\omega_{0}\right)$. The complementary part of $C\left(\omega_{0}\right)$ in $A\left(\omega_{0}\right)$ is denoted by $D\left(\omega_{0}\right)$. Without loss of generality we may assume that

$$
A\left(\omega_{0}\right)=\left(C\left(\omega_{0}\right) \mid D\left(\omega_{0}\right)\right) .
$$

For $x \in R^{s}$, we write $x=\left(x^{1}, x^{2}\right)$, where $x^{1}$ consists of the first $\nu$ components of $x$ and $x^{2}$ is the rest. Then

$$
Q_{J}\left(\omega_{0}\right)=\left\{x \in R^{s} \mid x^{1}=\left[C\left(\omega_{0}\right)\right]^{-1}\left(-\alpha\left(\omega_{0}\right)-D\left(\omega_{0}\right) x^{2}\right), x^{2} \in R^{s-\nu}\right\},
$$

where $\left[C\left(\omega_{0}\right)\right]^{-1}$ denotes the inverse matrix of $C\left(\omega_{0}\right)$ and $\alpha(\cdot)$ stands for the vector function whose components are $\alpha_{j}(\cdot), j \in J$. One can find a neighborhood $W$ of $\omega_{0}$ such that $C(\omega)$ is nonsingular whenever $\omega \in W$. Hence, we also have

$$
Q_{J}(\omega)=\left\{x \in R^{s} \mid x^{1}=[C(\omega)]^{-1}\left(-\alpha(\omega)-D(\omega) x^{2}\right), x^{2} \in R^{s-\nu}\right\} .
$$

From this we easily see that $Q_{J}(\cdot)$ is lower continuous on $W$. The lemma is proved.

Lemma 2.2. Suppose that the system $\left\{a_{j}(\omega), j \in J\right\}$ is linearly dependent for every $\omega$ on an open set $W_{0} \subseteq R^{r}$. Then there exist an open subset $W \subseteq W_{0}$ and a proper subset $J^{\prime} \subseteq J$ such that $\left\{a_{j}(\omega), j \in J^{\prime}\right\}$ is a maximal linearly independent subsystem of the system $\left\{a_{j}(\omega), j \in J\right\}$ for every $\omega \in W$.

Proof. For every $\omega \in W_{0}$, the rank of the system $\left\{a_{j}(\omega), j \in J\right\}$ cannot exceed $\nu-1$ where $\nu=|J|$, hence one can find a point $\omega_{0} \in W_{0}$ such that the rank of the system $\left\{a_{j}\left(\omega_{0}\right), j \in J\right\}$ is maximal on $W_{0}$ and equals, say $\mu<\nu$. Let $\left\{a_{j}\left(\omega_{0}\right), j \in J^{\prime}\right\}$ with $\left|J^{\prime}\right|=\mu, J^{\prime} \subseteq J$, be a linearly independent subsystem of the system $\left\{a_{j}\left(\omega_{0}\right), j \in J\right\}$. The matrix $A\left(\omega_{0}\right)$ whose rows are $a_{j}\left(\omega_{0}\right), j \in J^{\prime}$, possesses a nonsingular $(\mu \times \mu)$ submatrix, say $C\left(\omega_{0}\right)$. There exists, then, a neighborhood $W \subseteq W_{0}$ of $\omega_{0}$ such that $C(\omega)$ is nonsingular whenever $\omega \in W$. Hence, the subsystem $\left\{a_{j}(\omega), j \in J^{\prime}\right\}$ has rank equal to $\mu$, for every $\omega \in W$. In other words $\left\{a_{j}(\omega), j \in J\right\}$ is linear independent. It is maximal because the rank of the system $\left\{a_{j}(\omega), j \in J\right\}$ does not exceed $\mu$. The proof is complete.

Lemma 2.3. For every open set $W_{0} \subseteq R^{r}$ there exists an open subset $W \subseteq W_{0}$ such that the set-valued map $\omega \mapsto Q(\omega)$ is continuous on $W$.

Proof. If there exists a point $\omega_{0} \in W_{0}$ such that the system $\left\{a_{j}\left(\omega_{0}\right), j=p+\right.$ $1, \ldots, p+q\}$ is linearly independent, then in view of Lemma $2.1, Q$ is continuous on some neighborhood $W \subseteq W_{0}$ of $\omega_{0}$. If the system $\left\{a_{j}(\omega), j=p+1, \ldots, p+q\right\}$ 
is linearly dependent for every $\omega \in W_{0}$, we apply Lemma 2.2 to get an open subset $W_{1} \subseteq W_{0}$ and a proper subset $J \subseteq\{p+1, \ldots, p+q\}$ such that $\left\{a_{j}(\omega), j \in J\right\}$ is a maximal linearly independent subsystem of $\left\{a_{j}(\omega), j=p+1, \ldots, p+q\right\}, \omega \in W_{1}$. We claim that there is an open set $W \subseteq W_{1}$ such that either

$$
Q(\omega)=Q_{J}(\omega), \quad \text { for all } \omega \in W,
$$

or

$$
Q(\omega)=\emptyset, \quad \text { for all } \omega \in W .
$$

In fact, take an arbitrary index $j_{0} \in\{p+1, \ldots, p+q\} \backslash J$. If the system $\left\{a_{j}(\omega), j \in\right.$ $\left.J \cup\left\{j_{0}\right\}\right\}$ is linearly dependent, one can find $\lambda_{j}(\omega) \in R^{r}$ such that

$$
a_{j_{0}}(\omega)=\sum_{j \in J} \lambda_{j}(\omega) a_{j}(\omega)
$$

where $\lambda_{j}(\omega), j \in J$, are uniquely determined and continuous with respect to $\omega$ (because $\left\{a_{j}(\omega), j \in J\right\}$ is linearly independent) on some neighborhood of $\omega$. If at some $\omega \in W_{1}$ one has $\alpha_{j_{0}}(\omega) \neq \sum_{j \in J} \lambda_{j}(\omega) \alpha_{j}(\omega)$, then by the continuity of $\lambda_{j}(\cdot)$ and $\alpha_{j}(\cdot)$ there is a neighborhood $W \subseteq W_{1}$ of $\omega$ such that

$$
\alpha_{j_{0}}\left(\omega^{\prime}\right) \neq \sum_{j \in J} \lambda_{j}\left(\omega^{\prime}\right) \alpha_{j}\left(\omega^{\prime}\right), \quad \text { for all } \omega^{\prime} \in W .
$$

This shows that $Q\left(\omega^{\prime}\right)=\emptyset$ for every $\omega^{\prime} \in W$. If $\alpha_{j_{0}}(\omega)=\sum_{j \in J} \lambda_{j}(\omega) \alpha_{j}(\omega)$ for every $\omega \in W_{1}$, we have

$$
Q_{J}(\omega)=Q_{J \cup\left\{j_{0}\right\}}(\omega), \quad \omega \in W_{1} .
$$

Continuing this procedure for other indices from the set $\{p+1, \ldots, p+q\} \backslash J$, we arrive at the conclusion that either $Q(\omega)=\emptyset$ for all $\omega \in W$, or $Q(\omega)=Q_{J}(\omega)$ for all $\omega \in W$. In both cases, $Q$ is continuous on $W$.

Lemma 2.4. Given a point $\omega_{0} \in R^{r}$ and a subset $I \subseteq\{1, \ldots, p\}$. Assume that $\widehat{P}_{I}\left(\omega_{0}\right)$ is nonempty. Then, the map $\omega \mapsto P_{I}(\omega)$ is continuous on some neighborhood of $\omega_{0}$. Moreover, for every $x_{0} \in \widehat{P}_{I}\left(\omega_{0}\right)$ there exist a neighborhood $U$ of $x_{0}$ in $R^{s}$ and a neighborhood $W$ of $\omega_{0}$ in $R^{r}$ such that $U \subseteq \widehat{P}_{I}(\omega)$, for every $\omega \in W$.

Proof. As before, the closedness of $P_{I}$ is obvious. To prove the lemma it suffices to show the second part. We have

$$
\left\langle a_{i}\left(\omega_{0}\right), x_{0}\right\rangle+\alpha_{i}\left(\omega_{0}\right)<0, \quad \text { for all } i \in I .
$$

One can find a positive $\varepsilon$ such that

$$
\left\langle a_{i}\left(\omega_{0}\right)+a_{i}, x_{0}+x\right\rangle+\alpha_{i}\left(\omega_{0}\right)+\alpha_{i}<0, \quad \text { for all } i \in I,
$$

whenever $\left\|a_{i}\right\|<\varepsilon,\|x\|<\varepsilon,\left|\alpha_{i}\right|<\varepsilon$. By the continuity of $a_{i}(\cdot)$ and $\alpha_{i}(\cdot)$, there exists a neighborhood $W$ of $\omega_{0}$ such that

$$
\left\|a_{i}(\omega)-a_{i}\left(\omega_{0}\right)\right\|<\varepsilon, \quad\left|\alpha_{i}\left(\omega_{0}\right)-\alpha_{i}(\omega)\right|<\varepsilon,
$$

for every $\omega \in W$. Taking $U:=\left\{x \in R^{r} \mid\left\|x-x_{0}\right\|<\varepsilon\right\}$, we conclude that

$$
\left\langle a_{i}(\omega), x\right\rangle+\alpha_{i}(\omega)<0, \quad \text { for all } i \in I, \omega \in W, x \in U .
$$

This means that $U \subseteq \widehat{P}_{I}(\omega)$ for every $\omega \in W$, and the lemma is proved. 
Lemma 2.5. For every open set $W_{0}$, there exist an open subset $W \subseteq W_{0}$ and a subset $I \subseteq\{1, \ldots, p\}$ such that for every $\omega \in W$,

$$
P(\omega)=P_{I}(\omega) \cap Q_{J}(\omega), \quad \text { where } J:=\{1, \ldots, p\} \backslash I,
$$

and $\widehat{P}_{I}(\omega) \neq \emptyset$.

Proof. Observe first that $\widehat{P}_{i}(\omega)=\emptyset$ implies $\alpha_{i}(\omega)=0, a_{i}(\omega)=0$, and that $\widehat{P}_{i}\left(\omega_{0}\right) \neq$ $\emptyset$ for some $\omega_{0}$ implies $\widehat{P}_{i}(\omega) \neq \emptyset$ for every $\omega$ in a sufficiently small neighborhood of $\omega_{0}$. Hence without loss of generality we may assume that

$$
\widehat{P}_{i}(\omega) \neq \emptyset, \quad \text { for all } i \in\{1, \ldots, p\}, \text { for all } \omega \in W_{0} .
$$

Let $I(\omega)$ denote the maximal subset of $\{1, \ldots, p\}$ with $\widehat{P}_{I}(\omega) \neq \emptyset$ (with respect to the inclusion preorder), i.e.

$$
\widehat{P}_{I}(\omega) \cap \widehat{P}_{i}(\omega)=\emptyset, \quad \text { for all } i \in J .
$$

Since $|I(\omega)| \leq p$ for every $\omega \in R^{r}$, one can find a point $\omega_{0} \in W_{0}$ such that

$$
\left|I\left(\omega_{0}\right)\right|=\max \left\{|I(\omega)| \mid \omega \in W_{0}\right\} .
$$

We show that there is a neighborhood $W$ of $\omega_{0}$ such that $I(\omega)=I\left(\omega_{0}\right)$ for every $\omega \in W$. In fact, as in the proof of Lemma $2.4, \widehat{P}_{I\left(\omega_{0}\right)}\left(\omega_{0}\right) \neq \emptyset$ implies that there exists a neighborhood $W$ of $\omega_{0}$ such that $\widehat{P}_{I\left(\omega_{0}\right)}(\omega) \neq \emptyset$ for every $\omega \in W$. Hence $I\left(\omega_{0}\right) \subseteq I(\omega)$ whenever $\omega \in W$. We have, in fact, equality because $\left|I\left(\omega_{0}\right)\right|$ is maximal. Set $I=I\left(\omega_{0}\right)$. For every $\omega \in W$, one sees that

$$
P_{I}(\omega) \cap Q_{J}(\omega) \subseteq P(\omega) .
$$

On the other hand, $x \in P(\omega)$ implies

$$
\left\langle a_{j}(\omega), x\right\rangle+\alpha_{j}(\omega)=0, \quad j \in J .
$$

Indeed, if this is not the case, for some $j \in J$, one has

$$
\left\langle a_{j}(\omega), x\right\rangle+\alpha_{j}(\omega)<0 .
$$

Hence, there is a neighborhood $U$ of $x$ in $R^{s}$ such that

$$
\left\langle a_{j}(\omega), x^{\prime}\right\rangle+\alpha_{j}(\omega)<0, \quad \text { for all } x^{\prime} \in U .
$$

It is evident that $P_{I}(\omega)$ is the closure of $\widehat{P}_{I}(\omega)$ which is nonempty, hence one can find in $U$ some $x^{\prime} \in \widehat{P}_{I}(\omega)$. Consequently, $x^{\prime} \in \widehat{P}_{I}(\omega) \cap \widehat{P}_{J}(\omega)$ that contradicts the maximality of $I$. In this way, $P(\omega)=P_{I}(\omega) \cap Q_{J}(\omega)$, with $\widehat{P}_{I}(\omega) \neq \emptyset$ for every $\omega \in W$. This completes the proof.

Let $M_{1}, M_{2}$ be two set-valued maps from $R^{r}$ to $R^{s}$. It is known that $M_{1} \cap M_{2}$ is closed if $M_{1}$ and $M_{2}$ are closed, and that $M_{1} \cap M_{2}$ is not necessarily lower continuous if $M_{1}$ and $M_{2}$ are. The following exception is useful (see also [13]).

Lemma 2.6. Assume that $M_{1}$ is lower continuous at $\omega_{0} \in R^{r}$ and $M_{2}$ has the property that for every $x_{0}$ from a subset $M^{\prime} \subseteq M_{2}\left(\omega_{0}\right)$ one can find neighborhoods $W$ of $\omega_{0}$ and $U$ of $x_{0}$ such that $U \subseteq M_{2}(\omega)$ for every $\omega \in W$. Then the intersection map $M_{1} \cap M_{2}$ is lower continuous at $\omega_{0}$ provided that $M_{1}\left(\omega_{0}\right) \cap M_{2}\left(\omega_{0}\right)$ coincides with the closure of the set $M^{\prime} \cap M_{1}\left(\omega_{0}\right)$. 
Proof. Let $x \in M_{1}\left(\omega_{0}\right) \cap M_{2}\left(\omega_{0}\right)$, and let $U_{0}$ be any neighborhood of $x$ in $R^{s}$. We may find a point $x_{0} \in M^{\prime} \cap M_{1}\left(\omega_{0}\right)$ with $x_{0} \in U_{0}$. Then, there exist neighborhoods $U$ and $W$ as stated in the lemma. Take $U_{1}=U_{0} \cap U$. By the lower continuity of $M_{1}$, one can find a neighborhood $W_{1} \subseteq W$ of $\omega_{0}$ such that $M_{1}(\omega) \cap U_{1} \neq \emptyset$ for every $\omega \in W_{1}$. Consequently,

$$
\emptyset \neq U_{1} \cap M_{1}(\omega) \subseteq U_{0} \cap U \cap M_{1}(\omega) \subseteq U_{0} \cap\left[M_{1}(\omega) \cap M_{2}(\omega)\right]
$$

for every $\omega \in W_{1}$, and the lower continuity of $M_{1} \cap M_{2}$ follows.

Now we are ready to give

Proof of Proposition 2.1. We have $M(\omega)=P(\omega) \cap Q(\omega)$, for every $\omega \in R^{r}$. In view of Lemma 2.5, there exists an open subset $W_{1} \subseteq W_{0}$ such that $P(\omega)=$ $P_{I}(\omega) \cap Q_{J}(\omega), \omega \in W_{1}$, where $\widehat{P}_{I}(\omega) \neq \emptyset$ and $J=\{1, \ldots, p\} \backslash I$. Hence we may assume that $\widehat{P}(\omega) \neq \emptyset$ for all $\omega \in W_{1}$ (by considering $P_{I}$ instead of $P$ and $Q \cap Q_{J}$ instead of $Q$ ). By Lemma 2.3, there is a neighborhood $W \subseteq W_{1}$ such that $Q$ is continuous on $W$. Using Lemma 2.4 and Lemma 2.6, we conclude that $M$ is continuous on $W$.

\section{Continuity And DifFerentiability of the SOlution MAPPing}

In this section we study the marginal function $m$ and the solution map $S$ defined at the beginning of the previous section.

Proposition 3.1. Assume that $M$ is continuous on an open set $W_{0} \subseteq R^{r}$ and $M\left(\omega_{0}\right)$ is compact for some $\omega_{0} \in W_{0}$. Then there is a neighborhood $W \subseteq W_{0}$ of $\omega_{0}$ such that the marginal function is continuous on $W$.

Proof. It is evident that there exists a neighborhood $W \subseteq W_{0}$ of $\omega_{0}$ such that $M(\omega)$ is compact for $\omega \in W$. Direct verification shows that $m(\cdot)$ is continuous at every point $\omega \in W$ (see also [2], [4]).

Results similar to Proposition 3.1 can be found in several papers on marginal functions. In the rest of this note we shall prove a stronger result; namely, we shall show that it is possible to find a solution $\bar{x}(\omega)$ of the problem

$$
\min \{\langle c(\omega), x\rangle \mid x \in M(\omega)\}
$$

which depends smoothly on $\omega$ in $W$, provided that $c(\cdot), a_{i}(\cdot), \alpha_{i}(\cdot)$ are smooth. From now on, for the sake of simple presentation, we presume that $M(\omega)$ is compact for every $\omega$. A more general case will be treated in a forthcoming paper. Recall that the solution set is defined as

$$
S(\omega):=\{x \in M(\omega) \mid\langle c(\omega), x\rangle=m(\omega)\} .
$$

Let $I$ be any subset of $\{1, \ldots, p+q\}$. As before,

$$
Q_{I}(\omega):=\left\{x \in R^{s} \mid\left\langle a_{i}(\omega), x\right\rangle+\alpha_{i}(\omega)=0, i \in I\right\} .
$$

Lemma 3.1. Assume that the map $Q_{I} \cap M$ and the function $m(\cdot)$ are continuous on an open set $W_{0} \subseteq R^{r}$ and, for some $\omega_{0} \in W_{0}$,

$$
Q_{I}\left(\omega_{0}\right) \cap S\left(\omega_{0}\right)=\emptyset .
$$

Then, there exists a neighborhood $W \subseteq W_{0}$ of $\omega_{0}$ such that

$$
Q_{I}(\omega) \cap S(\omega)=\emptyset, \quad \text { for all } \omega \in W .
$$


Proof. Condition (1) implies that

$$
\left\langle c\left(\omega_{0}\right), x\right\rangle-m\left(\omega_{0}\right)>0, \quad \text { for all } x \in Q_{I}\left(\omega_{0}\right) \cap M\left(\omega_{0}\right) .
$$

Hence, there is $\varepsilon>0$ such that

$$
\left\langle c(\omega), x^{\prime}\right\rangle-m(\omega)>0
$$

provided

$$
\left\|c(\omega)-c\left(\omega_{0}\right)\right\|<\varepsilon, \quad\left|m(\omega)-m\left(\omega_{0}\right)\right|<\varepsilon,
$$

and

$$
\left\|x^{\prime}-x\right\|<\varepsilon
$$

for some $x \in Q_{I}\left(\omega_{0}\right) \cap M\left(\omega_{0}\right)$. The continuity of $c(\cdot)$ and $m(\cdot)$ implies the existence of a neighborhood $W_{1} \subseteq W_{0}$ of $\omega_{0}$ such that (4) holds whenever $\omega \in W_{1}$. The continuity of $Q_{I} \cap M$ shows that for some neighborhood $W \subseteq W_{1}$ of $\omega_{0}$,

$$
h\left(Q_{I}(\omega) \cap M(\omega), Q_{I}\left(\omega_{0}\right) \cap M\left(\omega_{0}\right)\right)<\varepsilon, \quad \text { for all } \omega \in W .
$$

This means that, for every $x^{\prime} \in Q_{I}(\omega) \cap M(\omega)$, one can find $x \in Q_{I}\left(\omega_{0}\right) \cap M\left(\omega_{0}\right)$ such that (5) holds. Consequently, (3) is satisfied whenever $\omega \in W$, and in fact (2) is true.

For every $\omega \in R^{r}$, denote by $V(\omega)=\left\{v_{i}(\omega), i \in\{1, \ldots, k(\omega)\}\right\}$ the set of all the vertices of $M(\omega)$ and by $J\left(v_{i}(\omega)\right)$ the index set of all equalities determining the vertex $v_{i}(\omega)$, i.e.

$$
\begin{gathered}
\left\langle a_{j}(\omega), v_{i}(\omega)\right\rangle+\alpha_{j}(\omega)=0, \quad j \in J\left(v_{i}(\omega)\right), \\
\left\langle a_{j^{\prime}}(\omega), v_{i}(\omega)\right\rangle+\alpha_{j^{\prime}}(\omega)<0, \quad j^{\prime} \in J^{c}\left(v_{i}(v)\right):=\{1, \ldots, p+q\} \backslash J\left(v_{i}(\omega)\right) .
\end{gathered}
$$

Lemma 3.2. For every open set $W_{0} \subseteq R^{r}$, there exists an open subset $W \subseteq W_{0}$ such that the cardinality of $V(\omega)$ (denoted by $k(\omega))$ is constant on $W$, say $k$. Moreover, the vertices of $M(\omega)$ can be indexed so that $J\left(v_{i}(\omega)\right)$ remains unchanged with respect to $\omega$ on $W$, for $i=1, \ldots, k$.

Proof. In view of Proposition 2.1, without loss of generality we may assume that $M$ is continuous on $W_{0}$. Since $p+q$ is fixed, the number of vertices of $M(\omega)$ is majorized by a fixed integer. Hence, there is a point $\omega_{0} \in W_{0}$ such that

$$
k\left(\omega_{0}\right)=\max \left\{k(\omega) \mid \omega \in W_{0}\right\} .
$$

We shall write $k:=k\left(\omega_{0}\right)$. Our aim now is to show that for every vertex $v_{i}\left(\omega_{0}\right)$ of $M\left(\omega_{0}\right)$ and for every $\delta>0$, there exist a neighborhood $W_{i} \subseteq W_{0}$ of $\omega_{0}$ and a positive $\varepsilon<\delta$ such that for every $\omega \in W_{i}$ one can find a vertex of $M(\omega)$, say $v_{i}(\omega)$, such that $\left\|v_{i}(\omega)-v_{i}\left(\omega_{0}\right)\right\|<\varepsilon$. In fact, if not, one can find a sequence $\left\{\omega_{n}\right\} \subseteq W_{0}$ converging to $\omega_{0}$ and a positive $\varepsilon_{0}$ such that

$$
\left\|v_{j}\left(\omega_{n}\right)-v_{i}\left(\omega_{0}\right)\right\| \geq \varepsilon_{0}, \quad \text { for all } j \in\left\{1, \ldots, k\left(\omega_{n}\right)\right\} .
$$

Since $M(\cdot)$ is continuous, there exists $x_{n} \in M\left(\omega_{n}\right)$ with $\lim _{n \rightarrow \infty} x_{n}=v_{i}\left(\omega_{0}\right)$. By (6), $x_{n}$ can be expressed as

$$
x_{n}=\sum_{j=1}^{k\left(\omega_{n}\right)} \lambda_{j}^{n} v_{j}\left(\omega_{n}\right)
$$

for some $\lambda_{j}^{n} \geq 0$ such that $\sum_{j} \lambda_{j}^{n}=1$. Since $k(\omega)$ is majorized, by taking a subsequence if necessary, we may assume that $\left\{v_{j}\left(\omega_{N}\right)\right\},\left\{\lambda_{j}^{n}\right\}$ converge respectively 
to $v_{j}^{0}, \lambda_{j}^{0}$. Again, the continuity of $M(\cdot)$ implies that $v_{j}^{0} \in M\left(\omega_{0}\right)$. In view of $(6)$ and (7),

$$
v_{i}\left(\omega_{0}\right)=\sum_{j} \lambda_{j}^{0} v_{j}^{0}
$$

with

$$
\left\|v_{j}^{0}-v_{i}\left(\omega_{0}\right)\right\| \geq \varepsilon_{0}, \quad \text { for all } j .
$$

It follows that $v_{i}\left(\omega_{0}\right)$ belongs to the convex hull of the points $v_{j}^{0}$ 's and $v_{i}\left(\omega_{0}\right) \neq v_{j}^{0}$, for all $j$. Therefore, $v_{i}\left(\omega_{0}\right)$ is not a vertex of this convex hull, which is contained in $M\left(\omega_{0}\right)$. This contradicts the fact that $v_{i}\left(\omega_{0}\right)$ is a vertex of $M\left(\omega_{0}\right)$. Now let us take $\delta>0$ so small that

$$
\left\|v_{i}\left(\omega_{0}\right)-v_{j}\left(\omega_{0}\right)\right\| \geq 4 \delta, \quad \text { for all } i, j \in\{1, \ldots, k\}, j \neq i .
$$

For this $\delta$, as before, we obtain $W_{1}, \ldots, W_{k}$ for the vertices $v_{1}\left(\omega_{0}\right), \ldots$, $v_{k}\left(\omega_{0}\right)$. We claim that $k(\omega)=k$ for every $\omega \in W^{\prime}:=W_{1} \cap \cdots \cap W_{k}$. In fact, for two arbitrary distinct vertices $v_{i}\left(\omega_{0}\right), v_{j}\left(\omega_{0}\right)$ of $M\left(\omega_{0}\right)$ and for every $\omega \in W^{\prime}$ one can find two vertices, denoted with the same indices $v_{i}(\omega), v_{j}(\omega)$, of $M(\omega)$ such that

$$
\left\|v_{i}(\omega)-v_{i}\left(\omega_{0}\right)\right\|<\delta, \quad\left\|v_{j}(\omega)-v_{j}\left(\omega_{0}\right)\right\|<\delta .
$$

One can see that $v_{i}(\omega) \neq v_{j}(\omega)$ because

$$
\begin{aligned}
\left\|v_{i}(\omega)-v_{j}(\omega)\right\| & \geq\left\|v_{i}\left(\omega_{0}\right)-v_{j}\left(\omega_{0}\right)\right\|-\left\|v_{i}(\omega)-v_{i}\left(\omega_{0}\right)\right\|-\left\|v_{j}(\omega)-v_{j}\left(\omega_{0}\right)\right\| \\
& \geq 4 \delta-2 \delta>\delta .
\end{aligned}
$$

Consequently, $M(\omega)$ has at least $k$ vertices, i.e. $k(\omega) \geq k$. Actually we have equality because $k$ is maximal. In sum, we have shown that for $\omega \in W^{\prime}, M(\omega)$ has exactly $k$ vertices $\left\{v_{1}(\omega), \ldots, v_{k}(\omega)\right\}$ which satisfy (8). For every fixed index $i \in\{1, \ldots, k\}$, the number $\left|J\left(v_{i}(\omega)\right)\right|$ is majorized by $p+q$. One can find a point $\omega_{*} \in W^{\prime}$ such that

$$
\left|J\left(v_{i}\left(\omega_{*}\right)\right)\right|=\min \left\{\left|J\left(v_{i}(\omega)\right)\right| \mid \omega \in W^{\prime}\right\} .
$$

We want to find a neighborhood $W_{i}^{\prime} \subseteq W^{\prime}$ of $\omega_{*}$ such that $J\left(v_{i}(\omega)\right)=J\left(v_{i}\left(\omega_{*}\right)\right)$, for all $\omega \in W_{i}^{\prime}$. In fact, if $j \notin J\left(v_{i}\left(\omega_{*}\right)\right)$, then

$$
\left\langle a_{j}\left(\omega_{*}\right), v_{i}\left(\omega_{*}\right)\right\rangle+\alpha_{j}\left(\omega_{*}\right)<0 .
$$

Since $a_{i}, \alpha_{i}$ are continuous, by (8) with $\delta$ sufficiently small, we can find a neighborhood $W_{j}^{\prime \prime} \subseteq W^{\prime}$ of $\omega_{*}$ such that

$$
\left\langle a_{j}(\omega), v_{i}(\omega)\right\rangle+\alpha_{j}(\omega)<0, \quad \text { for all } \omega \in W_{j}^{\prime \prime} .
$$

Take $W_{i}^{\prime}=\bigcap\left\{W_{j}^{\prime \prime} \mid j \in\{1, \ldots, p+q\} \backslash J\left(v_{i}\left(\omega_{*}\right)\right)\right\}$ to see that

$$
J\left(v_{i}(\omega)\right) \subseteq J\left(v_{i}\left(\omega_{*}\right)\right), \quad \text { for all } \omega \in W_{i}^{\prime} .
$$

Since $\left|J\left(v_{i}\left(\omega_{*}\right)\right)\right|$ is minimal, the inclusion becomes equality. By applying the above argument for $i=1,2, \ldots, k$, we arrive at an open subset $W=W_{k}^{\prime} \subseteq W_{k-1}^{\prime} \subseteq \cdots \subseteq$ $W_{1}^{\prime} \subseteq W^{\prime}$ which satisfies the requirements of the lemma.

Let us denote

$$
J_{0}(\omega):=\bigcup\left\{J\left(v_{i}(\omega)\right) \mid v_{i}(\omega) \in S(\omega)\right\}
$$


Lemma 3.3. For every open set $W_{0} \subseteq R^{r}$, there exists an open subset $W \subseteq W_{0}$ such that $J_{0}(\omega)$ remains unchanged for every $\omega \in W$.

Proof. Without loss of generality we may assume that $M$ as well as $Q_{I} \cap M$ are continuous on $W_{0}$ (where $I$ is any subset of $\{1, \ldots, p+q\}$ ). Let $\omega_{0} \in W_{0}$ be a point such that $\left|J_{0}\left(\omega_{0}\right)\right|=\min \left\{\left|J_{0}(\omega)\right| \mid \omega \in W_{0}\right\}$. Applying Lemma 3.1 for $I=\bigcup\left\{J\left(v_{j}\left(\omega_{0}\right)\right) \mid v_{j}\left(\omega_{0}\right) \notin S\left(\omega_{0}\right)\right\}$, one can find a neighborhood $W \subseteq W_{0}$ of $\omega_{0}$ such that

$$
J_{0}(\omega) \subseteq J_{0}\left(\omega_{0}\right), \quad \text { for all } \omega \in W .
$$

The minimality of $\left|J_{0}\left(\omega_{0}\right)\right|$ implies that $J_{0}(\omega)=J_{0}\left(\omega_{0}\right), \omega \in W$, and the proof is complete.

Proposition 3.2. Assume that $a_{i}(\cdot), \alpha_{i}(\cdot), c(\cdot)$ are differentiable. Then, for any open set $W_{0} \subseteq R^{r}$, there exists an open subset $W \subseteq W_{0}$ such that $S(\omega)$ admits a differentiable selection on $W$. Consequently, the set of nondifferentiability of $m(\cdot)$ is nowhere dense.

Proof. By Proposition 2.1, there exists an open set $W_{1} \subseteq W_{0}$ such that the maps $M$ and $Q_{I} \cap M$, where $I$ is any subset of $\{1, \ldots, p+q\}$, are continuous on $W_{1}$. In view of Lemma 3.3, $J_{0}(\omega)$ does not change on some open subset $W_{2} \subseteq W_{1}$. Let $W_{3} \subseteq W_{2}$ be an open set as stated in Lemma 3.2. We fix any point $\omega_{0} \in W_{3}$ and consider vertex $v_{i}\left(\omega_{0}\right)$ of $S\left(\omega_{0}\right)$. By Lemmas 3.2-3.3, for any $\omega \in W_{3}$, the solution of the system

$$
\left\langle a_{j}(\omega), x\right\rangle+\alpha_{j}(\omega)=0, \quad j \in J\left(v_{i}\left(\omega_{0}\right)\right)
$$

exists uniquely and is a vertex of $S(\omega)$. Let $A\left(\omega_{0}\right)$ be any nonsingular $(r \times r)$-matrix, whose columns are taken from $\left\{a_{j}\left(\omega_{0}\right), j \in J\left(v_{i}\left(\omega_{0}\right)\right)\right\}$. Then, $v_{i}\left(\omega_{0}\right)=\left[A\left(\omega_{0}\right)\right]^{-1}\left(-\alpha\left(\omega_{0}\right)\right)$, where $\alpha\left(\omega_{0}\right)$ is the vector of the values $\alpha_{j}\left(\omega_{0}\right)$ corresponding to the columns of $A\left(\omega_{0}\right)$. One can find a neighborhood $W \subseteq W_{3}$ of $\omega_{0}$ such that $A(\omega)$ is nonsingular whenever $\omega \in W$. It is evident that $v_{i}(\omega)=$ $[A(\omega)]^{-1}(-\alpha(\omega))$ and it is a differentiable selection of $S(\omega)$ on $W$. The marginal function $m(\omega))=\left\langle c(\omega), v_{i}(\omega)\right\rangle$, which is differentiable on $W$ as well. The proof is complete.

\section{ACKNOWLEDGMENT}

The authors wish to thank Professor J.-P. Penot of the University of Pau for valuable comments and suggestions on the first draft of the paper.

\section{REFERENCES}

1. J.-P. Aubin, Further properties of Lagrange multipliers in nonsmooth optimization, Appl. Math. Optim. 6 (1980), 79-90. MR 81a:90129

2. J.-P. Aubin and H. Frankowska, Set-valued analysis, Birkhauser, Basel, 1990. MR 91d:49001

3. B. Bank, J. Guddat, D. Klatte, B. Kummer, and K. Tammer, Nonlinear parametric optimization, Akademie-Verlag, Berlin, 1982. MR 84i:90147

4. C. Berge, Topological spaces, Macmillan, New York 1963.

5. G. B. Danzig, J. Folkman, and N. Shapiro, On the continuity of the minimum set of a continuous function, J. Math. Anal. Appl. 17 (1967), 519-548. MR 34:7241

6. P. H. Dien and D. T. Luc, On the calculation of a generalized gradient of a marginal function, Acta Math. Vietnam. 18 (1993), 309-326. MR 95f:49020

7. J. P. Evans and F. J. Gould, Stability in nonlinear programming, Oper. Res. 18 (1970), 107-118. MR 41:9573 
8. A. V. Fiacco, Introduction to sensitivity and stability analysis in nonlinear programming, Academic Press, New York, 1983. MR 85b:90072

9. J. Gauvin and F. Dubeau, Differential properties of the marginal function in mathematical programming, Math. Programming Study 19 (1982), 101-119. MR 84d:90099

10. J. B. Hiriart-Urruty, Gradients généralisés de fonctions marginales, SIAM J. Control Optim. 16 (1978), 301-316. MR 58:12599

11. K. Kuratowski, Topology, T. I+II Mir, Moscow, 1966. (Russian) MR 36:839

12. J. V. Outrata, On generalized gradients in optimization problems with set-valued constraints, Math. Oper. Res. 15 (1990), 626-639. MR 91k:49022

13. J.-P. Penot, Compact nets, filters and relations, J. Math. Anal. Appl. 93 (1983), 400-417. MR 84h:49032

14. _ Preservation of persistence and stability under intersection and operations, Parts I and II J. Optim. Theory Appl. (to appear). MR 94k:49013

15. S. M. Robinson Stability theory for systems of inequalities, Part I: Linear systems, SIAM J. Numer. Anal. 12 (1975), 754-769. MR 53:14270

16., A characterization of stability in linear programming, Oper. Res. 25 (1977), 435-447. MR 56:4815

17. R. T. Rockafellar. Lagrange multipliers and subderivatives of optimal value functions in nonlinear programming, Math. Programming Study 17 (1982), 28-66. MR 84h:90067

18. J. E. Spingarn, Fixed and variable constraints in sensitivity analysis, SIAM J. Control Optim. 18 (1980), 297-310. MR 81c:90084

19. L. Thibault, On subdifferentials of optimal value functions, SIAM J. Control Optim. 29 (1991), 1019-1036. MR 92e:49027

20. D. E. Ward, Differential stability in non-Lipschitzian optimization, J. Optim. Theory Appl. 73 (1992), 101-120. MR 93c:49011

21. N. D. Yen and P. H. Dien, On differential estimations for marginal functions in mathematical programming problems with inclusion constraints, Lectures Notes in Control and Inform. Sci. vol. 143, Springer-Verlag, Berlin, 1990, pp. 244-251.

Institute of Mathematics, P.O. Box 10000 Boho, Hanoi, Vietnam 